\title{
Ataques quânticos ao gerador pseudo-aleatório de Blum-Micali
}

\author{
Nigini A. Oliveira, Edmar J. Nascimento e F. M. Assis.
}

\begin{abstract}
Resumo-A utilização de seqüências pseudo-aleatórias é de grande importância em diversas aplicações da criptografia. Um ataque a um gerador pseudo-aleatório objetiva reproduzir uma dada seqüência pseudo-aleatória a partir de um conjunto de termos dessa seqüência. Classicamente, esse é um problema de difícil solução, ou seja, não existe algoritmos conhecidos em tempo polinomial que realizem esta tarefa. Por outro lado, o desenvolvimento de algoritmos quânticos vem mostrando que determinados tipos de problemas podem ser resolvidos de modo mais eficiente. Neste artigo, é apresentado um circuito quântico que realiza um ataque ao gerador Blum-Micali apresentando um ganho no número de operações em relação aos algoritmos clássicos conhecidos.
\end{abstract}

Palavras-Chave-Gerador Blum-Micali, algoritmos quânticos, sequiências pseudo-aleatórias.

Abstract-Pseudo-random sequences are very important to a wide range of applications in cryptography. The purpose of attacking a pseudo-random sequence is to predict it from a smaller set of terms. Classically, this is a problem that is hard to solve. This means that no polynomial-time algorithms are available. On the other hand, quantum algorithms are proved to solve some computation problems in a more efficient way. In this article, we present a quantum circuit that attacks BlumMicali's generator providing computational gains over classical counterpart solutions.

Keywords-Blum-Micali's generator, quantum algorithms, pseudo-random sequences.

\section{INTRODUÇÃO}

A utilização de seqüências de números aleatórios se faz necessária em diversas aplicações da criptografia tais como: esquemas de autenticação recíprocos, geração de chaves de sessão e a geração de chaves para o algoritmo de chave pública RSA [1]. As sequiências de números aleatórios devem atender a dois requisitos principais: aleatoriedade e imprevisibilidade. Para que a aleatoriedade seja garantida, os símbolos da seqüência devem ser uniformemente distribuídos e independentes. A imprevisibilidade garante que um termo da sequiência não pode ser inferido a partir de termos anteriores. Para aplicações práticas, é bastante difícil conseguir fontes de números verdadeiramente aleatórios. Dessa forma, recorrese a algoritmos determinísticos para a geração de seqüências que satisfazem alguns testes estatísticos. Essas sequiências são chamadas de pseudo-aleatórias.

Os geradores de seqüências pseudo-aleatórias atuam de modo bastante similar. Em geral, é realizado algum tipo de

Nigini A. Oliveira, Departamento de Sistemas e Computação. Edmar J. Nascimento e F. M. Assis, Departamento de Engenharia Elétrica. Universidade Federal de Campina Grande, Campina Grande-PB, Brasil, E-mails: nigini@dsc.ufcg.edu.br, jnedmar@dee.ufcg.edu.br, fmarcos@dee.ufcg.edu.br. Este trabalho foi parcialmente financiado pelo $\mathrm{CNPq}$ operação iterativa em aritmética modular, em que é usado como módulo um número primo muito grande. Dentre esses geradores, o gerador de Blum-Micali é um dos mais simples. A sua segurança é baseada na intratabilidade computacional do problema do logaritmo discreto [5]. Classicamente, a dificuldade desse problema parece ser da mesma ordem de magnitude que a fatoração de números primos requerida para o sistema RSA [1].

Por outro lado, existem algoritmos quânticos que se destacam na solução de problemas computacionais difíceis, que não possuem solução em tempo polinomial. Particularmente, os algoritmos de Grover, Shor, Deutsch e a transformada de Fourier quântica proporcionam ganhos consideráveis ao se comparar com os algoritmos clássicos correspondentes [2], [3]. Com a aplicação da transformada de Fourier quântica, consegue-se resolver o problema do logaritmo discreto com $\mathcal{O}(\lceil\log r\rceil)^{2}$ operações, em que $r$ é o menor número inteiro tal que $a^{r} \bmod p=1$. Uma das razões para o ganho computacional proporcionado pelos algoritmos quânticos é devido a uma propriedade conhecida como superposição quântica. A superposição quântica permite codificar todos os valores do domínio de uma dada função em um único estado quântico composto por qubits (bits quânticos). Ao realizar uma transformação sobre o estado quântico em superposição, realiza-se a avaliação simultânea de todos os valores do domínio da função a partir de uma única operação.

Motivado pelas boas perspectivas oriundas dos algoritmos quânticos, é proposto neste artigo um circuito quântico que permite encontrar a chave secreta do gerador Blum-Micali a partir da sequiência pseudo-aleatória gerada e dos parâmetros públicos do gerador. Esse circuito proporciona um ganho no número de operações realizadas. Este artigo está organizado da seguinte maneira. Na seção II, é descrito o funcionamento do gerador Blum-Micali e também são apresentados alguns resultados referentes à sua segurança. Na seção III, é apresentada uma revisão sobre alguns tópicos da mecânica quântica necessários ao entendimento da solução apresentada nesse artigo. Na seção IV, é apresentado o circuito quântico proposto nesse artigo para o ataque ao gerador Blum-Micali.

\section{Gerador De Blum-Micali}

O gerador Blum-Micali (BM) é definido da seguinte forma [7]. Seja $p$ um número primo, escolhe-se $g$ como sendo um gerador para $\mathbb{Z}_{p}^{*}$ e a semente, a chave secreta do gerador, $x_{0}$. Os valores de $p$ e $g$ podem ser publicamente conhecidos e usados diversas vezes seguidas. A geração dos bits da sequiência pseudo-aleatória é feita da seguinte forma: para o 
$i$-ésimo bit $b_{i}$, começando com $i=1$, tem-se:

$$
\begin{aligned}
x_{i} & =g^{x_{i-1}} \bmod p ; \\
b_{i} & =\delta_{x_{i}>(p-1) / 2} .
\end{aligned}
$$

Em que a equação (2) indica que $b_{i}=1$ se e somente se $x_{i}>(p-1) / 2$.

A prova de que o gerador BM é computacionalmente seguro é baseada na hipótese da intratabilidade computacional do problema do logaritmo discreto. Blum e Micali mostraram que admitindo-se a hipótese precedente, não é possível calcular em tempo polinomial o bit $b_{i}$ a partir do valor de $x_{i+1}$. Esse problema é conhecido como hard-core bit problem.

\section{TÓPICOS GERAIS DA MECÂNICA QUÂNTICA}

Os sistemas quânticos são descritos pelas leis da mecânica quântica e esta, por sua vez, se baseia num conjunto de postulados que servem de base para toda a teoria quântica [4]. Um sistema quântico isolado é descrito por um vetor de estados unitário $|\psi\rangle$ em um espaço de Hilbert $\mathcal{H}$. O sistema quântico mais simples é o qubit (bit quântico), um sistema quântico associado a um espaço de Hilbert de dimensão dois. De acordo com a notação de Dirac, um qubit pode ser representado como

$$
|\psi\rangle=a|0\rangle+b|1\rangle
$$

Sendo que $|0\rangle=\left[\begin{array}{ll}1 & 0\end{array}\right]^{T}$ e $|1\rangle=\left[\begin{array}{ll}0 & 1\end{array}\right]^{T}$ formam uma base ortonormal para o espaço de estados do sistema conhecida como a base computacional. $a$ e $b$ são números complexos arbitrários que satisfazem a relação de normalização $|a|^{2}+$ $|b|^{2}=1$. A equação (3) pode ser interpretada como uma superposição dos estados $|0\rangle$ e $|1\rangle$, em que esses estados tem respectivamente as probabilidades $|a|^{2}$ e $|b|^{2}$ de serem observados após uma medida de $|\psi\rangle$ na base computacional.

A evolução de sistemas quânticos isolados é descrita por meio de transformações unitárias, de forma que o vetor de estados $|\psi\rangle$ no instante de tempo $t_{1}$ está relacionado com o vetor de estados $\left|\psi^{\prime}\right\rangle$ no instante de tempo $t_{2}$ pela relação

$$
\left|\psi^{\prime}\right\rangle=U|\psi\rangle \text {. }
$$

Sendo que $U$ é um operador unitário que depende apenas dos instantes $t_{1}$ e $t_{2}$.

As medidas quânticas são descritas através de observáveis. Um observável é uma propriedade de um sistema físico que, em princípio, pode ser medida como por exemplo, a posição de uma partícula. Na mecânica quântica, um observável é representado por um operador auto-adjunto $\mathbf{A}$, em que $\mathbf{A}=$ $\mathbf{A}^{\dagger}$. Um operador auto-adjunto em um espaço de Hilbert $\mathcal{H}$ possui uma representação espectral, ou seja, os seus autoestados formam uma base ortonormal completa em $\mathcal{H}$. Um operador auto-adjunto $\mathbf{A}$ pode ser então expressado como

$$
\mathbf{A}=\sum_{n} \lambda_{n} \mathbf{P}_{n}
$$

Em que $\lambda_{n}$ é um autovalor de $\mathbf{A}$ e $\mathbf{P}_{n}$ é um projetor ortogonal no espaço dos autovetores com autovalor $\lambda_{n}$. Ao medir um sistema quântico, a saída numérica do processo de medição de um observável A é um autovalor $\lambda_{n}$ de $\mathbf{A}$. O estado do sistema é modificado, de modo que logo após a medição, o estado quântico passa a ser um auto-estado do observável A correspondente ao autovalor medido. Se o estado quântico imediatamente antes da medida é $|\psi\rangle$, então a saída $\lambda_{n}$ é obtida com probabilidade

$$
P\left(\lambda_{n}\right)=\| \mathbf{P}_{n}|\psi\rangle \|^{2}=\left\langle\psi\left|\mathbf{P}_{n}\right| \psi\right\rangle
$$

Se a saída $\lambda_{n}$ é obtida, o estado normalizado pós-medição é

$$
\frac{\mathbf{P}_{n}|\psi\rangle}{\sqrt{\left\langle\psi\left|\mathbf{P}_{n}\right| \psi\right\rangle}} \text {. }
$$

A medida do estado $|\psi\rangle$ dado pela equação (3) com o observável $A=|0\rangle\langle 0|-| 1\rangle\langle 1|$ resulta em +1 com probabilidade $|a|^{2}$ e -1 com probabilidade $|b|^{2}$. Se o resultado da medida for +1 , o estado pós-medição é $|0\rangle$. Se o resultado da medida for -1 , o estado pós-medição é $|1\rangle$.

\section{A. Circuitos Quânticos}

Os algoritmos quânticos são implementados através de circuitos quânticos. Nos circuitos quânticos, os qubits são os elementos de informação sobre os quais são realizadas operações a fim de obter um resultado desejado. As transformações realizadas sobre os qubits são descritas por meio de transformações unitárias implementadas pelas portas quânticas. Uma porta quântica de grande importância é a porta de Hadamard. A porta de Hadamard é descrita pela operação unitária

$$
H \equiv \frac{1}{\sqrt{2}}\left[\begin{array}{cc}
1 & 1 \\
1 & -1
\end{array}\right]
$$

Ao atuar nos estados da base computacional $|0\rangle$ e $|1\rangle$, a porta de Hadamard realiza as seguintes transformações

$$
\begin{aligned}
& |0\rangle \quad \stackrel{H}{\rightarrow} \frac{1}{\sqrt{2}}(|0\rangle+|1\rangle) \equiv|+\rangle, \\
& |1\rangle \quad \stackrel{H}{\rightarrow} \frac{1}{\sqrt{2}}(|0\rangle-|1\rangle) \equiv|-\rangle .
\end{aligned}
$$

Ou seja, a sua aplicação cria uma superposição de estados a partir dos estados base $|0\rangle$ e $|1\rangle$. A aplicação de portas de Hadamard nos qubits individuais de um sistema composto de $N$ qubits gera uma superposição de $2^{N}$ termos. Por exemplo, para o estado $|\psi\rangle=|0\rangle^{\otimes N}$, a aplicação de $H^{\otimes N}$ resulta em

$$
H^{\otimes N}|0\rangle^{\otimes N}=\frac{1}{2^{n / 2}} \sum_{x_{i} \in\{0,1\}^{n}}\left|x_{i}\right\rangle .
$$

A superposição gerada pela equação (11) permite gerar a entrada para circuitos quânticos mais complicados como o que é proposto neste artigo.

\section{B. Algoritmo de Grover}

O algoritmo de Grover [8], ou algoritmo quântico de busca, utiliza-se da superposição descrita pela equação (11) para realizar a busca de $M$ elementos em um conjunto desordenado de $N$ elementos. Para isso, é necessário a construção de uma transformação unitária denominada oráculo $(O)$. Esta porta é responsável por marcar os elementos buscados dentro do conjunto completo de elementos, que por sua vez estão codificados em uma superposição. 
Um exemplo dessa operação é a busca do elemento 1 no conjunto $\{0,1,2,3\}$, o qual pode ser codificado através de uma superposição de dois qubits. Para este exemplo, o estado inicial do sistema pode ser representado pelo estado $|\Psi\rangle=|001\rangle$, em que os dois primeiros qubits são usados para codificar os quatro valores possíveis do espaço de busca e o terceiro é utilizado pelo oráculo de Grover para a marcação do elemento buscado. A aplicação do operador Hadamard (8) em cada um dos três qubits de $|\Psi\rangle$ cria um estado $\left|\Psi_{1}\right\rangle=$ $1 / 2(|00\rangle+|01\rangle+|10\rangle+|11\rangle) \otimes|-\rangle$. É sobre este estado que o oráculo deve ser aplicado. $\mathrm{O}$ oráculo necessário para efetuar a marcação do valor 1 no estado $\left|\Psi_{1}\right\rangle$ é obtido através de uma porta quântica CNOT (NOT controlado) no circuito quântico indicado na Figura 1. A aplicação desse circuito inverte a fase do terceiro qubit, transforma $|-\rangle$ em $|+\rangle$, apenas para o termo |01〉 da superposição.

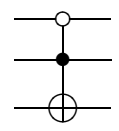

Fig. 1. Circuito quântico para a marcação do elemento 1 codificado pelo elemento $|01\rangle$ da superposição.

O mais importante a se entender é que o resultado desta operação inverte, a grosso modo, o sinal do elemento procurado. Um engano comum é pensar que a leitura dos qubits neste ponto resulta no elemento marcado. Na verdade, o elemento marcado teve apenas uma inversão de fase e não de sua amplitude probabilística com relação ao restante dos elementos do conjunto. Por isso, uma terceira e última fase é necessária ao algoritmo de Grover, a amplificação de fase realizada pela transformação $2|\Psi\rangle\langle\Psi|$. A amplificação de fase é responsável por aumentar a probabilidade de leitura daqueles valores que receberam a marcação.

Para uma maior probabilidade de leitura dos elementos marcados, a aplicação do oráculo seguida pela amplificação deve ser executada algumas vezes. Essa seqüência iterativa é chamada de iteração de Grover $(G=2|\Psi\rangle\langle\Psi| O)$ e a quantidade de vezes que ela deve ser executada para levar a maior probabilidade possível depende apenas de $M$ e $N$. Vários estudos já foram feitos a respeito e prova-se que a quantidade de iterações é da ordem de $\mathcal{O}(\sqrt{N / M})$ [9].

\section{BUSCA QUÂNTICA DE $X_{i}$}

Nesta seção são analisadas algumas características de um ataque quântico ao gerador BM descrito na seção II. A idéia base do algoritmo é a reconstrução da palavra gerada $b$, testando as possíveis seqüências geradoras, partindo-se do conjunto com todos os possíveis $x_{0}$ e reduzindo-se o mesmo levando em consideração os bits $b_{i}$. Obviamente que no caso clássico esta solução força-bruta não leva a bons resultados devido à explosão exponencial de possibilidades. No caso da utilização de um computador quântico, pode-se lançar mão do seu paralelismo para sanar tal dificuldade.

Nas análises de segurança, pressupõe-se que o atacante do gerador BM tem conhecimento dos seguintes parâmetros:

- O módulo primo $p$;
- O gerador do grupo finito $g$;

- Uma palavra pseudo-aleatória $b=b_{1} b_{2} \cdots b_{l}$ gerada pelo gerador BM.

O circuito de ataque proposto é mostrado na Figura 2. Esse circuito possui como entrada quatro registradores e os seus blocos estão divididos em quatro fases de processamento. O primeiro registrador possui os bits $b_{i}$ da palavra gerada, codificados através dos estados quânticos $\left|b_{i}\right\rangle$, enquanto o segundo possui $\lceil\log p\rceil$ qubits, necessários para gerar os elementos $x_{i}$ calculados pela equação (1). O terceiro e o quarto registradores são compostos por qubits auxiliares, sendo que o quarto possui apenas um qubit utilizado pelo algoritmo de Grover e o terceiro recebe tantos qubits quantos $b_{i}$ s forem utilizados no primeiro registrador.

As quatro etapas do circuito realizam as seguintes funções:

1) Preparação da superposição a partir dos qubits do segundo registrador com portas Hadamard;

2) Iterações de filtragem (portas $F\left(b_{i}\right)$ ) e permutação (portas $P$ ) dos elementos do segundo registrador, marcando o resultado no terceiro registrador;

3) Aplicação de $\sqrt{p}$ iterações de Grover (porta $G^{*}$ ), para amplificar o elemento marcado;

4) Leitura dos qubits do segundo registrador.

A seguir, a segunda e a quarta etapa do circuito são detalhadas.

\section{A. Segunda Etapa - Filtragem e Permutação}

Esta segunda etapa do algoritmo simula o ataque clássico força-bruta no que diz respeito a aplicar o algoritmo de BlumMicali em todos os elementos do grupo. A diferença clara é que a utilização da superposição quântica torna estes cálculos muito mais eficientes. Assim como o gerador pseudo-aleatório, o circuito mostrado é iterativo, buscando filtrar, dentro do conjunto de possibilidades, aqueles elementos capazes de gerar o bit $b_{i}$ em questão.

Esta etapa do circuito é montada com dois tipos de porta. $F\left(b_{i}\right)$ é um filtro dos elementos que podem ter gerado o bit $b_{i}$ segundo o algoritmo de Blum-Micali. Assim, a função desta porta é apenas testar se os elementos da superposição são maiores ou menores que $l=(p-1) / 2$. Caso $b_{i}$ seja 0 e o elemento superposto menor que 1 , então um bit específico no terceiro registrador será invertido. $\mathrm{O}$ mesmo bit será invertido no caso em que $b_{i}$ seja 1 e o elemento maior que $l$.

A segunda porta usada nesta fase é denominada $P$. Esta opera apenas sobre o segundo registrador, porém, controlada pelos elementos do terceiro. A operação realizada é a permutação dos elementos marcados simulando a geração de cada bit da palavra $b_{i}$. Uma permutação nos moldes do algoritmo de BM pode ser implementado por um somatório de projetores.

\section{B. Quarta Etapa - Amplificação e leitura}

Esta etapa do algoritmo é composta pela aplicação do algoritmo de Grover já explicada na seção III-B. Para isso, o oráculo a ser utilizado no circuito proposto como solução é definido pelo circuito indicado a seguir: 


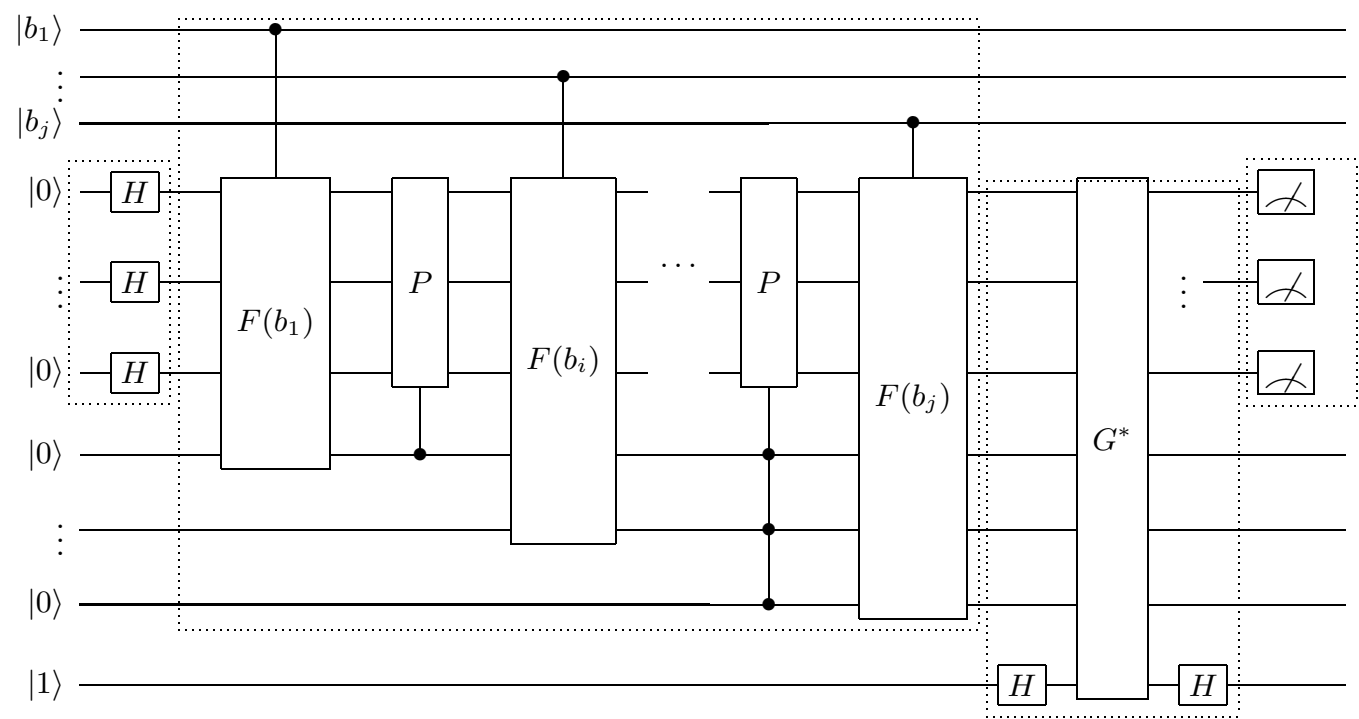

Fig. 2. Circuito de ataque ao gerador BM. As quatro fases do circuito estão destacadas nos retângulos tracejados da direita para a esquerda.

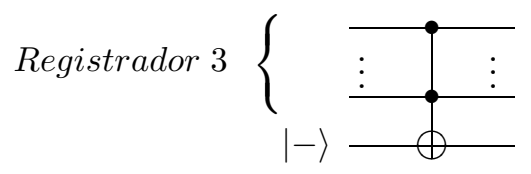

Significando que o elemento do segundo registrador, associado ao valor $|11 \ldots 1\rangle$ no terceiro registrador terá sua probabilidade de leitura amplificada a um valor muito próximo de 1 .

Como já definido anteriormente, o algoritmo quântico de busca executa $\mathcal{O}(\sqrt{N / M})$ chamadas ao oráculo, sendo que no caso do circuito proposto, $M=1$ e $N=\log p$. Desta forma, o circuito será capaz de ler um valor $x_{i}$, com alta probabilidade, após $\mathcal{O}(\sqrt{\log p})$ chamadas ao oráculo.

Com a aplicação desse algoritmo, o gerador BM tem um estado interno $x_{i}$ identificado a partir de valores disponíveis a qualquer atacante. Com este valor, é possível simular deterministicamente o funcionamento do gerador, quebrando a propriedade de pseudo-aleatoriedade do mesmo. Um último passo pode ainda ser realizado para a descoberta do valor da chave secreta $x_{0}$. Para isso é utilizado o cálculo do logaritmo discreto, que é um problema tratável através da transformada quântica de Fourier (ver seção I).

\section{Exemplos}

1) Exemplo 1: Considera-se um gerador BM definido por $p=7$ e $g=3$. A sequiência binária gerada pelo gerador é $b=10$. Desta forma o circuito apresentado necessita de dois qubits no primeiro registrador, $\lceil\log 7\rceil=3$ no segundo para representar os números de um a sete em binário, e mais três qubits auxiliares. A entrada do circuito pode ser escrita no estado $|\Psi\rangle=|10\rangle \otimes|000\rangle \otimes|00\rangle \otimes|1\rangle$. Após a aplicação das portas Hadamard na primeira fase do circuito, o estado inicial do algoritmo sem normalização é descrito por:

$$
\begin{aligned}
\left|\Psi_{0}\right\rangle= & |10\rangle \otimes(|000\rangle+|001\rangle+|010\rangle+|011\rangle+ \\
& |100\rangle+|101\rangle+|110\rangle+|111\rangle) \otimes \\
& |00\rangle \otimes|1\rangle
\end{aligned}
$$

A aplicação da operação de filtragem a partir do primeiro qubit $\left|b_{1}\right\rangle=|1\rangle$ no estado $\left|\Psi_{0}\right\rangle$ resulta em:

$$
\begin{aligned}
\left|\Psi_{1}\right\rangle= & F(1)\left|\Psi_{0}\right\rangle=|10\rangle \otimes \\
& {[(|000\rangle+|001\rangle+|010\rangle+|011\rangle)|00\rangle)+} \\
& (|100\rangle+|101\rangle+|110\rangle+|111\rangle)|10\rangle] \otimes|1\rangle
\end{aligned}
$$

. Observa-se então que os elementos do segundo registrador que são maiores que $l=(7-1) / 2=3$ foram associados ao estado $|10\rangle$ no terceiro registrador. Leva-se em consideração aqui, o fato de que a implementação de um circuito clássico para o teste maior-que-menor-que é trivial e eficiente. Assim, a implementação da porta $F$ através de um circuito quântico é provadamente possível e eficiente, como mostrado em [10].

A operação de permutação é obtida a partir da tabela de permutação do grupo $\mathbb{Z}_{p}^{*}$. Para os estados que não estão nesse grupo, a permutação leva esses estados para eles mesmos. Sendo assim, $P$ é dado por (qubits representados em notação decimal):

$$
\begin{aligned}
P= & |0\rangle\langle 0|+| 5\rangle\langle 1|+| 2\rangle\langle 2|+| 6\rangle\langle 3|+ \\
& |4\rangle\langle 4|+| 5\rangle\langle 5|+| 1\rangle\langle 6|+| 7\rangle\langle 7| .
\end{aligned}
$$

A aplicação de $P$ em $\left|\Psi_{1}\right\rangle$ leva o sistema ao estado $\left|\Psi_{2}\right\rangle$ dado por:

$$
\begin{aligned}
\left|\Psi_{2}\right\rangle= & P\left|\Psi_{1}\right\rangle=|10\rangle \otimes \\
& {[(|000\rangle+|001\rangle+|010\rangle+|011\rangle)|00\rangle)+} \\
& (|100\rangle+|101\rangle+|001\rangle+|111\rangle)|10\rangle] \otimes|1\rangle .
\end{aligned}
$$

Neste caso, apenas o elemento $|110\rangle$ é permutado para o valor $|001\rangle$, pois o operador $P$ é controlado pelo primeiro qubit do terceiro registrador e as outras componentes $(|100\rangle$ e $|101\rangle)$ são permutadas para o mesmo valor. Aplicando novamente a operação de filtragem, agora levando em conta o qubit $\left|b_{2}\right\rangle=$ 
$|0\rangle$, tem-se o novo estado do sistema:

$$
\begin{aligned}
\left|\Psi_{3}\right\rangle= & F(0)\left|\Psi_{2}\right\rangle=|10\rangle \otimes \\
& {[(|000\rangle+|001\rangle+|010\rangle+|011\rangle)|00\rangle)+} \\
& (|100\rangle+|101\rangle+|111\rangle)|10\rangle+ \\
& (|001\rangle)|11\rangle] \otimes|1\rangle .
\end{aligned}
$$

Percebe-se que um único elemento está associado ao valor $|11\rangle$ no terceiro registrador, significando que a etapa de filtragem foi concluída.

2) Exemplo 2: Considera-se agora que o gerador BM é definido por $p=19$ e $g=2$ e a seqüência binária é dada por $b=b_{1} b_{2} b_{3}=101$. Como a quantidade de qubits no segundo registrador é $k=\lceil\log p\rceil=5$, a superposição gerada pelas portas Hadamard compreende a faixa de valores $\{0,1, \cdots, 31\}$. Porém, os números válidos para esse gerador $\mathrm{BM}$ pertencem à faixa $\{1,2, \cdots, 18\}$. Esses valores presentes no estado $\left|\Psi_{0}\right\rangle$ são eliminados pelas próprias iterações de execução do circuito apresentado, ou ainda, pode-se considerar um pequeno passo de marcação (similar à porta $F$ ) que elimina todos os valores maiores que $p-1$. Além disso, deve-se também considerar a necessidade de construir a porta $P$ de forma que possua a projeção de todos os valores do conjunto: $|19\rangle\langle 19|+\ldots+| 32\rangle\langle 32|$, para que o operador seja unitário.

$\mathrm{Na}$ execução do algoritmo, o teste relativo ao bit $b_{1}$ marcará o grupo de elementos $\{10, \ldots, 18,19, \ldots, 32\}$, pois apenas estes elementos são maiores que $l=9$. A porta $P$ permuta estes elementos e gera um novo sub-conjunto igual a $\{5,3,6,1,10,12,15,11,17,19, \ldots, 32\}$. O teste para o segundo bit marcará apenas os valores $\{5,3,6,1\}$, eliminando os números indesejados do conjunto inicial. Após isso seguese outra permutação baseada agora nos dois primeiros bits de marcação do terceiro registrador gerando $\{13,8,7,2\}$. Como o terceiro bit a ser testado é igual a 1 , o teste marca apenas o número $\{13\}$.

\section{Desempenho do Algoritmo}

A principal questão neste algoritmo é relacionada a quantidade de bits de $b_{i}$ necessários para a filtragem de um único elemento do grupo. No caso do segundo exemplo, quando a palavra é $b=101$, apenas três iterações são necessárias, mas se caso o terceiro bit fosse zero, o algoritmo necessitaria de mais algumas iterações. A hipótese levada em consideração neste trabalho é a de que o algoritmo de Blum-Micali utiliza-se de grupos e geradores que distribuem o melhor possível os elementos quando estes são permutados. Desta forma a filtragem reduzirá, em média, o número de elementos pela metade a cada passo. Esta estimativa define uma curva exponencial de redução, exigindo uma quantidade de iterações de filtragem da ordem de $\mathcal{O}(\log p)$ passos.

\section{CONClusões}

$\mathrm{O}$ circuito proposto nesse artigo realiza um ataque ao gerador BM em $\log p \sqrt{\log p}$ passos. Duas linhas de investigação estão sendo seguidas para a melhoria dos resultados obtidos. A primeira está relacionada a uma melhor análise da estrutura do grupo finito que define a estrutura de permutação e filtragem utilizada pela solução proposta. Os autores estão analisando os resultados apresentados em [11] a fim de melhorar a estimativa da quantidade de bits de $b$ necessários a uma filtragem perfeita.

Uma segunda linha relaciona-se a real eliminação dos termos da superposição que não satisfazem o bit $b_{i}$ antes da etapa de permutação. $O$ fato de eliminar esses termos anula a necessidade da utilização do algoritmo de Grover no final da execução. Nessa linha, os autores estão analisando a possibilidade de utilizar um resultado proposto em [6], o lema da amplificação. Esse lema estabelece que sob determinadas condições, é possível para um circuito $Q$ indicado na Figura 3 , construir um circuito $R$ que obtenha a saída $|0\rangle \otimes\left|\phi_{0}\right\rangle$ no circuito $Q$ em no máximo $(1 / p) \log 1 / \varepsilon$ iterações com probabilidade de no mínimo $1-\varepsilon$. Com isso, poderia-se por exemplo eliminar com alta probabilidade todos os termos da superposição que possuem o primeiro qubit $|1\rangle$ quando o bit $b_{i}$ correspondente fosse igual a 0 .

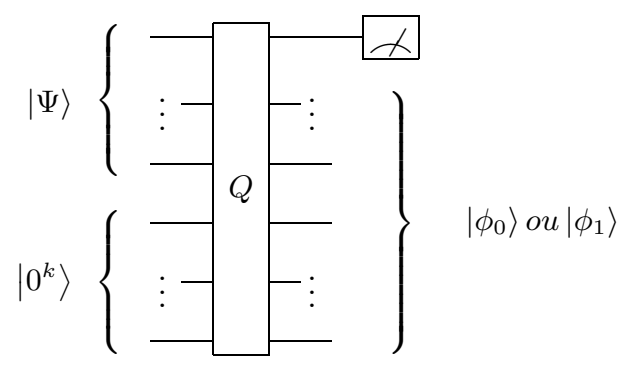

Fig. 3. O circuito $Q$ possui como entrada um estado $|\psi\rangle \otimes\left|0^{\otimes k}\right\rangle$. A sua saída consiste em $|0\rangle \otimes\left|\phi_{0}\right\rangle$ ou $|1\rangle \otimes\left|\phi_{1}\right\rangle$.

\section{Agradecimentos}

Os autores agradecem aos órgãos de fomento CAPES e $\mathrm{CNPq}$ pelo suporte financeiro ao projeto IQuanta(CTINFRA/FINEP 01.04.0061.00). Agradecem também aos membros do IQuanta Bernardo Lula, Aércio de Lima e Cheyenne Ribeiro pelas valiosas discussões acerca desse trabalho.

\section{REFERÊNCIAS}

[1] W. Stallings, Cryptography and Network Security: Principles and Practice. Prentice-Hall, Inc., New Jersey, 2 edition, 1998.

[2] Michael A. Nielsen and Isaac L. Chuang, Quantum Computation and Quantum Information. Cambridge University Press, 2000.

[3] Dirk Bouwmeester, Artur Ekert, and Anton Zeilinger, The Physics of Quantum Information. Springer, 2000.

[4] Leslie E. Ballentine, Quantum Mechanics - A Modern Development. World Scientific, 1998.

[5] R. A. C. Medeiros, Protocolo para Autenticação Quântica de Mensagens Clássicas. Dissertação de Mestrado, UFCG, 2004.

[6] John Watrous, Zero-Knowledge Against Quantum Attacks. Proceedings of the 38th ACM Symposium on Theory of Computing (STOC'06), 296-305, USA, 2006.

[7] Andrey Sidorenko and Berry Schoenmakers, State Recovery Attacks on Pseudorandom Generators. Proceedings of the Western European Workshop on Research in Cryptology (WEWoRC 2005), 53-64, LeuvenHeverlee, Belgium, 2005.

[8] Lov K. Grover, A fast quantum mechanical algorithm for database search. Proceedings of 28th ACM Symposium on Theory of Computing (STOC'1996), 212-219, 1996.

[9] Michel Boyer, Gilles Brassard, Peter Hoeyer, and Alain Tapp, Tight bounds on quantum searching. In Fortschritte der Physik, v. 46, p. 493, 1998.

[10] Charles Bennett, Logical Reversibility of Computation. In IBM Journal of Research and Development, v. 17, p. 525, 1973.

[11] Daniel R. Cloutier, Mapping the Discrete Logarithm. Dissertação de Mestrado, Rose-Hulman Institute of Techonology, 2005. 